\title{
Der Basler Stadtarzt Johannes Huber (1507-1571)*
}

\author{
Von Marie-Louise Portmann
}

Sechs Jahre nach dem Eintritt Basels in den Schweizerbund wurde der spätere Stadtarzt Johannes Huber 1507 als ältester Sohn von Martin Huber (1460-ca. 1544), dem Wirt zum Bock, geboren. Der Vater war während des Schwabenkriegs aus Ravensburg in Basel eingewandert und hatte 1504 das Basler Bürgerrecht erworben ${ }^{1}$. In die Jugendzeit Johannes Hubers fiel die Blüte des Humanismus in der Rheinstadt, denn von 1514 bis 1516 hielt sich Erasmus von Rotterdam das erstemal in Basel auf, wo er bei dem Drucker Johannes Froben die erste Ausgabe des Neuen Testaments in griechischer Sprache herausgab (1516). Der begabte Knabe erhielt schon früh den Anfangsunterricht in den alten Sprachen; doch die Eltern schickten ihn bald nach Schlettstadt, wo es eine renommierte Lateinschule gab, die von zahlreichen Baslern absolviert wurde ${ }^{2}$. Seit 1508 unterrichtete dort Johann Witz, genannt Sapidus (1490-1561), der bald der Leiter der Schule wurde ${ }^{3}$. Huber genoß dort seinen Unterricht wie auch der achtzehnjährige Thomas Platter, der sich dort «under die kleinnen kind» setzte, um endlich etwas Rechtes zu lernen. Platter schreibt: «was äben wie ein gluggerin under den hünlinen»... «Das war die erst schull, do mich ducht, das recht zu ging. Zu der zyt giengen die studia und linguae uff; ist in dem jar gsin, do der richstag zu Wurms ist gsin. Sapidus hatt eins mals 900 discipulos, ettlich fin glerte gsellen; do was do zumall doctor Hier. Gemusaeus, doctor Johannes Huberus und sunst vill ander, die sidhar doctores und verriempte menner worden sind.» ${ }^{4}$

Nach seiner Rückkehr nach Basel immatrikulierte sich Huber am 13. August 1521 an der Universität Basel ${ }^{5}$. Als Glarean (1488-1563) von Paris zurückkehrte (1522), wurde Huber ein Schüler dieses berühmten Humanisten und poeta laureatus aus Mollis, der sich durch die Kenntnis der alten Sprachen auszeichnete und auch die Geschichte, Geographie, Mathematik und Musik beherrschte ${ }^{6}$. Wohl von Glarean beeinflußt, begab sich Huber 1525 nach Paris, wo er an der Sorbonne die philosophische Magisterwürde erwarb. Er entschloß sich dann, Medizin zu studieren, und begab sich

* Zu dieser Arbeit angeregt wurde ich durch Herrn Dr.phil. Bernhard Prijs, Basel.

Gesnerus 1/2 (1981) 
zu diesem Zweck nach Montpellier, später nach Toulouse, wo er wegen seiner Gelehrsamkeit und seiner Kenntnis der griechischen Sprache zum Rektor der Universität gewählt wurde. Nachdem er den Doktorgrad erworben hatte, kehrte er 1536 nach Basel zurück, wo er alsbald zu praktizieren begann ${ }^{7}$. Damit hatte er bald solchen Erfolg, daß er nicht nur von den Baslern, sondern vielfach auch von den Adligen aus der Region konsultiert wurde. Auch Kollegen und auswärtige Ärzte zogen ihn gerne zu einem Consilium zu.

Seine erste Frau, Barbara Brand, die ihm drei Kinder gebar, verstarb jung. Am 2. Mai 1541 heiratete er dann Margret Wölfflin (1522-1579), von der er zwölf Kinder hatte. Sein ältester Sohn, Martin (1536-1564), wurde 1564 Professor der Jurisprudenz an der Universität Basel, starb aber noch im gleichen Jahr an der Pest. Ein weiterer Sohn, Hans Rudolf (1545-1601), wurde 1594 Bürgermeister von Basel, und dessen Bruder Hans Wernhart (1555-1587) wurde Doktor der Medizin ${ }^{8}$.

Noch bevor Johannes Huber Professor war, übte er bereits Funktionen an der Universität aus. Vom 1. Mai 1543 bis zum 30. April 1544 war er nämlich Rektor ${ }^{9}$. Als sein bereits erwähnter Schulkamerad Hieronymus Gemusaeus (1505-1544) starb, wurde Huber als dessen Nachfolger Professor der Physik an der Artistenfakultät ${ }^{10}$. Doch in die Rektoratszeit Hubers fällt ein bedeutendes Ereignis in der Geschichte der Medizin. Andreas Vesal war nach Basel gekommen, um bei dem Drucker Oporin sein epochemachendes Werk «De humani corporis fabrica» herauszubringen. Am 12. Mai 1543 hielt Vesal eine öffentliche Anatomie an der Leiche eines Missetäters, bei der sicher auch Johannes Huber zugegen war, wenn dies auch nicht aktenmäßig belegt werden kann. Über Beziehungen zwischen Vesal und Huber wissen wir nichts. Aus dem Taufbuch von St. Martin ist lediglich zu ersehen, daß Vesal am 3. August 1543 Pate war bei der Taufe eines Kindes Ruprecht Winters, wobei der siebenjährige Sohn Hubers, Martin, die Stellvertretung des andern Paten, Johann Rudolf Stoer von Stoerenburg (1499-1570), übernahm ${ }^{11}$, der 1542 bis 1570 Abt von Murbach und Luders war und der Johannes Huber zu seinem Leibarzt gewählt hatte. Bei einer andern Taufe vertrat der letztere selbst den Abt als Paten. Es war dies im Februar 1545, als das Töchterlein Placidia des Chronisten Johannes Gast (gest. 1552) zu St. Martin getauft wurde. Gast trug dieses Ereignis in sein Diarium ein ${ }^{12}$. Er und Oporin waren durch Freundschaft und Patenschaft mit Huber eng verbunden. Gast taufte zahlreiche Kinder Hubers zu St. Martin. Oporin war Pate Martins, des ältesten Sohnes des Arztes ${ }^{13}$. Huber wohnte auch in der 


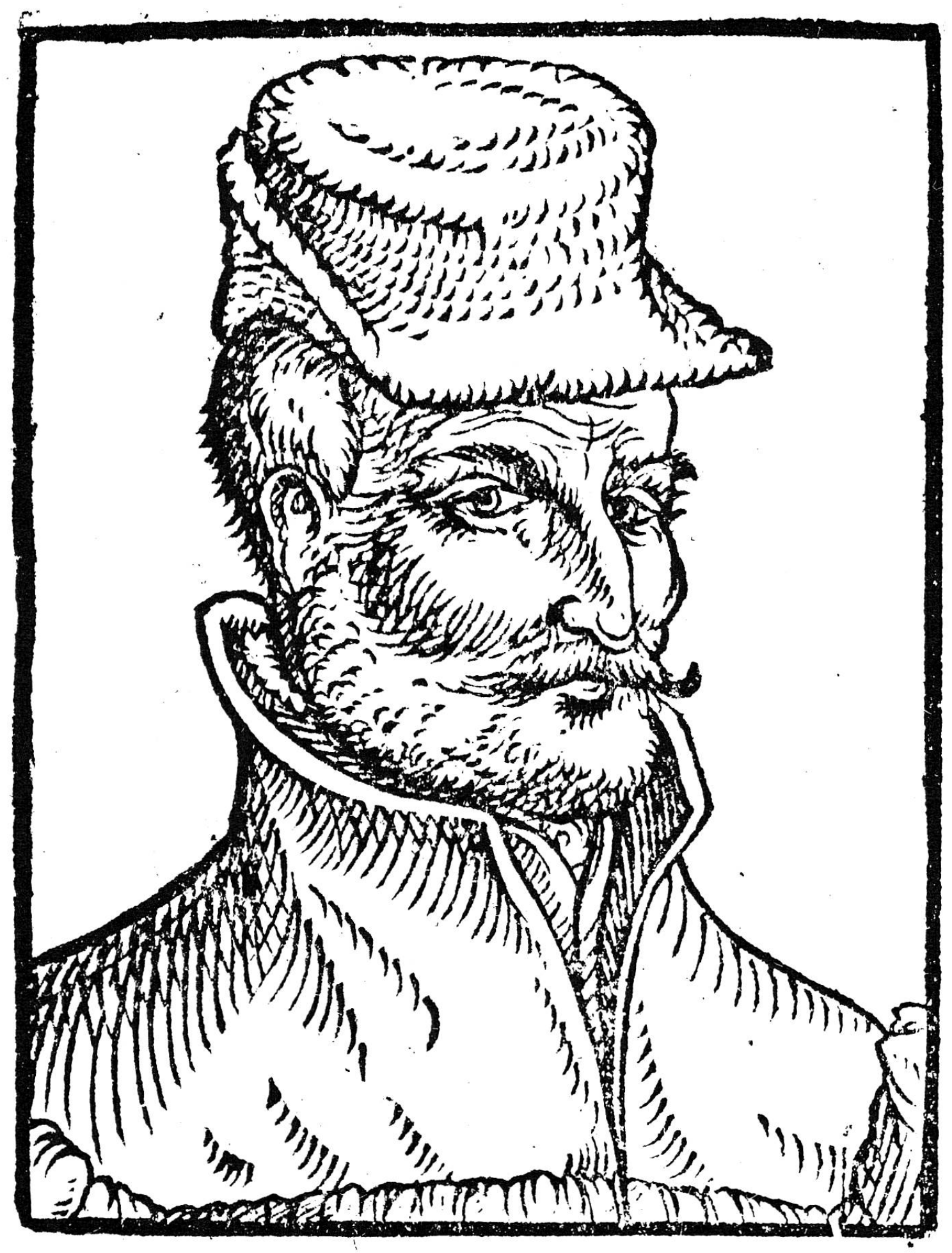

Johannes Huber 
Pfarrei St.Martin, denn seit dem 4.Oktober 1540 besaß er das Haus «zum hohen Sunnenlufft», Augustinergasse $1{ }^{14}$.

Huber muß ein besonders leutseliger, umgänglicher Mensch gewesen sein, schreibt doch sein Kollege Heinrich Pantaleon (1522-1595) über ihn: «Denn zu dieser Gelehrsamkeit kamen hinzu seine Redlichkeit, seine Heiterkeit und seine Willfährigkeit, allen Rechtschaffenen zu Diensten zu sein. Daher lebte er mit den Frommen fromm, mit den Klugen klug und mit den Scherzenden scherzend, so daß er mit Recht ein Mann für alle Stunden genannt werden konnte.» ${ }^{15}$

Nach dem Tode des Arztes Sebastian Sinckeler 1547 wurde Huber von dem einflußreichen Professor der Jurisprudenz, Bonifatius Amerbach (1495-1562), konsultiert. Aus dieser Zeit ist uns ein Brief Hubers erhalten, den er an seinen Patienten schrieb ${ }^{16}$. Daraus ist zu ersehen, daß Huber seine Diagnose durch Harnschau stellte. Er glaubte auf diese Weise zu bemerken, daß die Leber Amerbachs affiziert sei, was vom Reiten herrühre. Als Heilmittel rät er, morgens und abends je eine Portion haltbar gemachter Zichorie einzunehmen. Dazu empfiehlt er Kichererbsen, denn er habe beobachtet, daß durch den Harn viel Sand abgegangen sei. Huber schreibt weiter: «Worinn ich mim herren kan lieben dienst bewysen, soll er mich nytt sparen.»

1546 war Huber in das Consilium Medicorum aufgenommen worden, ein Ärztegremium, das dem Dekan der Medizinischen Fakultät helfend zur Seite stand und bei der Erstellung von Gutachten mitwirkte ${ }^{17}$. Als dann im Jahre 1552 Oswald Bär (1482-1567) zu dozieren aufhörte, sollte ein Nachfolger bestellt werden. Da setzte sich der angesehene Zunftmeister und Ratsherr Jakob Rüedin (1501-1573) kräftig ein für Huber, den Gatten seiner Stieftochter. Er gab Bonifatius Amerbach zu bedenken, «jr wellenn uch ein Bassler lieber lossen sinn dann ein frembdenn, und helffen und Ratten, dass dockter Hanss nitt hindann gesetz werdt, sunders gefürdertt». ${ }^{18}$ Am 4. Juli 1552 stellten dann Bürgermeister Theodor Brand und der Rat eine Urkunde ${ }^{19}$ aus, die besagt, daß Huber «zu unser statt arzetten unnd der schulen ordinarien der arzny syn läben lang, unnd so lang er zu läsenn vermögenlich und togenlich, angenommen und bestellt» worden sei. Huber wurde zur Auflage gemacht, die Stadt nicht zu verlassen, um den Einwohnern stets zur Verfügung zu stehen; doch durfte er Fremde behandeln, wenn sie nach Basel kamen. Ausnahmen galten nur für Jakob Philipp von Gundelsheim (gest. 1553), den Bischof von Basel, sowie für Johann Rudolf Stoer, von dem schon die Rede war. Es war Huber gestattet, als Leibarzt dieser geistlichen 
Herren die Stadt zu verlassen. Doch mußte er für die Dauer seiner Abwesenheit einen Stellvertreter bestellen, der seine Vorlesungen an der Universität weiterhielt. Als Besoldung bekam Huber «zu anfang zu einer fügenn verehrung fünffzig pfundt stebler unnd demnoch jerlich und eins jeden jars besonders zwey hundert pfundt stebler unser stett werung». Für den Fall, daß er nicht mehr lesen könne, wurde ihm eine Chorherrenpfründe zu St. Peter in Aussicht gestellt.

Von Urlaub ist in der Bestellungsurkunde nicht die Rede; doch wissen wir aus andern Quellen, daß Huber sich alljährlich bei einer Badekur in Baden zu vergnügen pflegte. In einem aus Baden vom 3. Juli 1558 datierten Brief an Bonifatius Amerbach ${ }^{20}$ empfiehlt er dem Basler Juristen den kaiserlichen Rat Leonhard von Berkenstein, der zusammen mit Huber gebadet hatte und ihn um dieses Empfehlungsschreiben bat. Ein weiterer Brief aus Baden vom 28. Juni $1568{ }^{21}$ ist an seinen Sohn Johann Rudolf gerichtet, der gerade sein Haus umbaute, was der Vater billigte und wofür er seinem Sohn auch Geld anbot. Dem Großvater Rüedin schickte Huber «hundert kreps», die er anscheinend bekommen hatte, denn in Baden wurde gut getafelt, wir wir gleich sehen werden. Seinen Töchtern Esther (sechsjährig) und Agnes (fünfjährig) sandte der Arzt als Spielzeug «ein Lädlin/do sinn zwei iesus knäblin». Ferner muß Johann Rudolf einen Brief des Zürcher Magistrats an die Basler Regierung weiterleiten. Alsdann meldet Huber freudig, was er alles von Gönnern und Bekannten an Eßwaren bekommen habe: zwei Faß Wein, einen Zuber Butter, Fisch und Fleisch von Königsfelden, ferner Brot. Das Baden, Essen und Trinken sei gegenwärtig die Hauptsache. Nur zwei Tage später, am 30. Juni $1568{ }^{22}$, empfiehlt Huber den Junker Jacob von Landenberg an Theodor Zwinger (1533-1588) weiter. Dieser wurde von dem kaiserlichen Leibarzt Johannes Crato von Krafftheim (1519-1585) gebeten, den Junker zu behandeln. Landenberg hatte sich in Zips (Landschaft der Slowakei) im Kriegslager aufgehalten, wo eine mit Kopfweh verbundene Epidemie grassiert hatte. Da Landenberg nachher ständig von Kopfschmerzen geplagt war, badete er zuerst in Göppingen, dann in Baden, wo Huber seine Kunst an ihm versuchte, was aber auch nicht half. Huber meldet Zwinger, Crato habe Landenberg auf die «Holzordnung» gewiesen, womit die Anwendung des Lignum Guajaci gemeint war. Dies, meint Huber, sei das letzte Rettungsmittel. Von sich selbst meldet Huber, daß die Frauen gerne baden, essen und trinken. Doch der Basler Arzt würde sich langweilen, wenn er nicht so viele Geschäfte hätte und praktizieren könnte. Ein Hauptmann Adam aus Wien schrieb Huber, daß das ihm verordnete Tränklein wohlge- 
tan habe; doch klagt er immer noch über «Lemme (Lähmung) der Schencklen». Huber riet dem Patienten, sich in ein Bad zu begeben. Auch seinem Kollegen Zwinger meldet er voll Freude, welche Eßwaren er zugeschickt erhalten hat; doch ist er eigentlich froh, daß die Hälfte seines Aufenthaltes in Baden bereits vorbei ist. Während Hubers Abwesenheit hatten die Professoren Isaac Keller und Heinrich Pantaleon die Vorlesungen an der Universität anstelle Hubers übernommen und ihn vertreten.

Wie ernst Huber seine Pflichten als Arzt nahm und sich gedrängt fühlte, persönlich bei seinen Patienten anwesend zu sein, wenn es schlimm um sie stand, ersehen wir aus einem undatierten Brief Hubers ${ }^{23}$ an Johann Jacob Grynaeus (1540-1617), worin er sich entschuldigt, daß er bei ihm nicht erscheinen kann. Die Frau seines Schwagers Wernher Wölfflin ist krank geworden, so daß es ihm «von jren zuo wijchen nytt müglich ist». Überdies ist ein Enkel Jakob Rüedins, an dem dieser Vaterstelle vertritt, da der Sobn bereits gestorben ist, am «roten Schaden» erkrankt (Ruhr). Da kann Huber auch nicht weg, «denn der guott herr (Rüedin) hatt die kijnder gar lieb». Mit einem Stoßseufzer schließt Huber den Brief: «Ego sum ad labores natus. muos, also min leben lang, gefrettet und geplagt sijn. ist mir kein fröwd bescheiden.»

Daß Huber sich bei der Ausübung der Heilkunst nicht auf die Harnschau beschränkte, sondern mit den Ärzten seiner Zeit ernsthaft mitdiskutierte, geht eindeutig aus einem Brief an den Schaffhauser Arzt Johann Cosmas Holzach (1518-1595) ${ }^{24}$ vom 28. Mai 1570 hervor. Holzach hatte Huber um Rat bei der Behandlung eines wassersüchtigen Junkers gefragt. In seiner Antwort ${ }^{25}$ erwähnt der Basler Arzt, daß er oft Wassersucht zu behandeln habe. Er empfiehlt dafür vor allem zwei Mittel: erstens einen Syrupus Magistralis, der harntreibend und stuhlfördernd wirke. Als Ingredienzien enthielt er u.a. Zichorie, Fenchel, Spargel, Calamus, Sennesblätter, Anis, Zimt, Sandalum und Rhabarber. Das Rezept stammte von Eucharius Holzach (1486-1558), der Arzt in Basel und der Vater des Ratsuchenden war. Zweitens empfiehlt Huber einen Umschlag aus pulverisierter Wolfsleber und ausgewählten Heilkräutern, der auf den Magen und die Leber zu applizieren sei.

In seiner Heilkunst bewegte sich Huber in durchaus konservativen Bahnen; doch trug er insofern entscheidend zum Fortschritt der Medizin bei, als er es war, der den jungen Felix Platter (1536-1614) begeisterte und ihm als treuer Förderer zur Seite stand. Dies soll im folgenden skizziert werden. Schon als Knabe bewunderte Platter diejenigen Ärzte, die in sammetver- 
brämten scharlachroten Mänteln einhergingen, wie denn er selber, der spätere Stadtarzt, sein Leben lang größte Sorgfalt auf ein gediegenes Äußeres verwendete. Bei Huber beeindruckte ihn vor allem der Umstand, daß dieser ausritt und einen Bediensteten als Vorreiter hatte ${ }^{26}$. Platters aufkeimende Liebe zu der Chirurgentochter Magdalena Jeckelmann vertraute er zuerst seinem Busenfreund Martin Huber an, dem ältesten Sohn des Stadtarztes, der von 1550 bis 1553 im Hause von Thomas Platter als Zögling wohnte ${ }^{27} .1552$ immatrikulierte sich Felix an der Universität Basel, wo gerade Johannes Huber zu dozieren begonnen hatte und vorübergehend beide Lehrstühle, sowohl den der praktischen wie auch den der theoretischen Medizin, versehen zu haben scheint. Platter hörte bei Huber eine Vorlesung über die hippokratische Schrift «De natura hominis» ${ }^{28}$. Als sich dann Platter zum weiteren Studium nach Montpellier begeben hatte, stand er in regem Briefwechsel sowohl mit Johannes als auch mit Martin Huber. Dieser Briefwechsel ist leider verlorengegangen ${ }^{29}$. Huber war es, der schon längst vor Platters Rückkehr dessen künftigen Ruhm voraussagte und die Erwartungen der Basler wachhielt. In seinem Tagebuch erwähnt Felix Platter, was ihm sein Vater schrieb: «Sagt, D. Huber sage allenthalben, ich werde ein firtreffenlicher artzet werden, hör er von andren und merck es aus meinen briefen .... ${ }^{30}$ Später erwähnt Felix einen andern Brief seines Vaters: «Erzelt auch, dass vil doctores by inen syen, haben aber wenig ausserthalb $\mathrm{D}$. Huber zeschaffen; derselbig sage vil guts von mir, ich werde in ersetzen, hab er vor den heupteren in einem pancket gesagt, auch vor meim künftigen schwecher und seiner dochter ... ${ }^{31}$ Im Frühjahr 1557 kehrte Felix Platter nach Basel zurück in Begleitung von Hans Jakob Rüedin d.J. (1538-1564). Dieser Reisegefährte gebärdete sich anmaßend gegenüber Platter und zog ihn auf, wie er wohl gegen die Konkurrenz des berühmten Doktor Hans aufkommen könne ${ }^{32}$. Doch dieser begrüßte den Heimkehrer schon am zweiten Tag nach seiner Ankunft in Basel auf das herzlichste. Felix schenkte Huber ein schön gebundenes Exemplar der Gedichte des französischen Dichters Clément Marot (1496-1544) ${ }^{33}$.

Bei der Vorbereitung der Hochzeit Platters mit Magdalena Jeckelmann spielte Huber, von Vater Thomas gebeten, eine Hauptrolle als Brautwerber. Felix schreibt: Nachher kam Huber «mit frolocken, wie sein bruch war, mir verkünte, glick wunste, doch vermeldet, ess begerte mein schwecher, dass die sach stil verblibe, bis der doctorat firüber, alss dan kente man die sach zu ende bringen, dessen ich wol zefriden ${ }^{34}$

Es sei hier einschaltend bemerkt, daß Huber später während der Brautzeit 
des Basilius Amerbach (1534-1591) eine ähnliche Rolle spielte. Am 24. März $1560^{35}$ dankt Huber für einen Brief Amerbachs, der zu Schiff heil in Speyer angekommen ist. Der Stadtarzt meldet, wie glücklich die Braut über diesen Brief gewesen sei, so daß sie ihn sogar geküßt habe. Sie bat Huber, wiederum an Amerbach zu schreiben und ihn in ihrem Namen zu grüßen, was Huber versprach. Der Arzt erwähnt ferner, daß er oft mit der Braut über Basilius spreche. Der Vater, Oberstzunftmeister Rüedin, sieht das gerne und läßt Basilius ebenfalls grüßen. Es scheint auch, daß Huber den Studenten Basilius und später den Professor der Jurisprudenz ärztlich betreut hat. In einem undatierten Brief ${ }^{36}$ an seinen Patienten, der sich in Nürnberg aufhielt, gesteht Huber zu, daß ein gewisser Heiltrank ausgesetzt werden könne bis nach Amerbachs Rückkehr. Ferner zeigt sich Huber erfreut, daß der Schleim reichlich aus Amerbachs Nase fließe, denn der Schleim sei der allgemeine Feind der Gelehrten. Es sei gut, wenn das Gehirn sich dadurch entlaste. Nach der Viersäftelehre glaubte man, das Gehirn sei bei einer Erkältung voll Schleim. Huber sieht keine Ursache für Amerbach, die unternommene Reise zu unterbrechen.

Doch kehren wir zu Felix Platter zurück, der nach erfolgter Brautwerbung erst noch sein Doktorexamen in Basel abzulegen hatte, wobei auch Huber als Mitglied des Collegium medicum mitzureden hatte. Die Hauptrolle spielte allerdings Oswald Bär, der den Titel und das Amt eines Professors und Stadtarztes beibehalten hatte, auch nachdem Huber 1552 diese Funktionen übernommen hatte. Bär war es auch, der Platter nach bestandenem Examen die Insignien eines Doktors der Medizin überreichte.

Bei den letzten Eheverhandlungen zwischen Thomas Platter und Franz Jeckelmann, den Vätern der Brautleute, nahm Huber teil unter den Vertrauten Platters ${ }^{37}$. Auch an der Hochzeit war Huber anwesend. Er hätte Brautführer sein sollen, mußte sich aber wegen eines Todesfalls in der Familie entschuldigen. Platter bemerkt in seinem Tagebuch: «kam doch uf die hochzeit, do er auch dantzt.» ${ }^{38} 1558$ nahmen Platter und Huber an der Hochzeit des Jakob Myconius (ca.1530-1559), des Stadtarztes in Mülhausen, teil, die am 1. Mai gefeiert wurde. Platter bemerkt: «... und kam zum nachtessen D. Hans Huber, der auch geladen und on dass zum apt von Murbach, den Stören, riten wolt.» ${ }^{39}$

Später scheint Platter, was die Art und Weise des Praktizierens betrifft, mehr und mehr in eine kritische Distanz zu Huber geraten zu sein. So ist auch Platters Erzählung, wie der Abt Johann Rudolf Stoer von Stoerenburg seines Leibarzt Huber mit Platter vertauschte, recht vorsichtig zu genießen, 
wie bereits der Herausgeber von Platters Tagebuch, Valentin Lötscher, richtig bemerkt hat. Daß Huber den Abt in der Krankheit verlassen und dessen Wappen «ins heimlich ort solt gesetzt haben», ja daß Huber nachher den Abt kniefällig um Verzeihung gebeten habe, ist unwahrscheinlich ${ }^{40}$. Doch wissen wir, daß Platter auch Patienten Hubers mitbehandelte, so zum Beispiel den Stoffel Schwertfeger, der 1566 nach Thann wegzog, wo sein Sohn Syndicus wurde. In diesem Zusammenhang macht Platter die abfällige Bemerkung: «da trieb Dr. Hans seine possen mit dem wasserbeschauwen.» ${ }^{41}$ Platter war moderner und maß der Harnschau keine Bedeutung mehr bei.

Johannes Huber war dreimal Rektor der Universität, 1543/44, 1555/56 und $1567 / 68^{42}$. Als solcher hatte er oft illustre Gäste zu begrüßen. So wurden Alexander Secundus und Philipp Eduard Fugger 1556 auf dem Wege zum Studium in Dôle in Basel von Rektor Huber und den Dekanen bewirtet ${ }^{43}$. In einem undatierten Brief an Amerbach ${ }^{44}$ lädt Huber diesen ein zu einem Nachtmahl mit Hans-Jakob Fugger, dem Herrn von Mörsberg und Doktor Peutingers Sohn. In die letzte Amtszeit Hubers als Rektor (1567) fallen die Bemühungen der Basler, den ehemaligen Sekretär des Erasmus, Gilbert Cousin (1506-1572), freizubekommen, der wegen seiner Neigungen zum reformierten Glauben in Dôle eingekerkert worden war. Cousin hatte Jurisprudenz, Medizin und Theologie studiert und war seit 1535 Kanonikus zu Nozeroy, wo er Basler Knaben bei sich aufnahm und unterrichtete ${ }^{45}$. Einer seiner Zöglinge war 1563 Johann Rudolf Huber ${ }^{46}$, ein Sohn des Medizinprofessors. Die Basler Demarchen ${ }^{47}$ bei Claude de la Baume, dem Erzbischof von Besançon, und bei den Behörden der Stadt Dôle scheinen zunächst von Erfolg gekrönt gewesen zu sein, denn Cousin wurde freigelassen. Er wurde aber später aufs neue inhaftiert und starb $1572 \mathrm{im} \mathrm{Gefängnis.}$

Johannes Huber scheint nichts publiziert zu haben. Sein Kollege Heinrich Pantaleon spricht 1565 davon, daß Huber an einem Buch arbeite, das sich auf die Heilkunst und die Erfahrungen des Basler Arztes in der Behandlung von Krankheiten beziehe. Pantaleon spricht die Hoffnung aus, daß das Buch bald erscheine ${ }^{48}$. Doch ist es wahrscheinlich, daß der Verfasser starb, während er noch an dem Werke arbeitete.

Wie wir gesehen haben, war Huber eine zentrale Gestalt im öffentlichen und wissenschaftlichen Leben Basels. Seine Liebenswürdigkeit machte ihn zu einem gesuchten Arzt, der durch seine Menschlichkeit dazu beitrug, daß die Mängel im Stand der damaligen ärztlichen Wissenschaft seinen Zeitgenossen nicht so sehr bewußt wurden. 


\section{Anmerkungen}

${ }^{1}$ Pantaleon, Heinrich, Prosopographiae heroum atque illustrium virorum totius Germaniae Pars tertia, Basel 1565, S.445.

2 Ebenda.

3 Die Amerbachkorrespondenz, hrsg. A. Hartmann, Bd. I, Basel 1942, S. 368.

${ }^{4}$ Platter, Thomas, Lebensbeschreibung, hrsg. A. Hartmann, Basel 1944, S. 56.

${ }^{5}$ Die Matrikel der Universität Basel, hrsg. H. G. Wackernagel, Bd.I, 1460-1529, Basel 1951, S.347.

${ }^{6}$ Pantaleon a.a. 0.

7 Ebenda.

${ }^{8}$ Wappenbuch der Stadt Basel, hrsg. W. R.Staehelin, Basel o. J. (1817 ff.), Stammbäume.

${ }^{9}$ Die Matrikel der Universität Basel, hrsg. H.G.Wackernagel, M.Sieber und H.Sutter, Bd. II, 1532/33-1600/01, Basel 1956, S.34.

10 Pantaleon a.a. O.

${ }^{11}$ Roth, Moritz, Andreas Vesalius Bruxellensis, Berlin 1892, S.129, Anm. 3.

${ }^{12}$ Basler Chroniken, Bd. 8, Das Tagebuch des Johannes Gast, Basel 1945, S. 217 ff.

13 Ebenda S.87.

14 Platter, Felix, Tagebuch, hrsg. V. Lötscher, Basel/Stuttgart 1976, S. 309, Anm. 34.

${ }^{15}$ Pantaleon a.a.O. «Nam ad hanc eius eruditionem accedebat vitae integritas, morum comitas, et omnibus bonis morigerendi voluntas. Itaque cum piis pie, cum prudentibus prudenter, cum iocantibus iocose vivebat, ut merito omnium horarum homo dici potuisset.»

${ }^{16}$ Brief Hubers an Bonifatius Amerbach vom 29. Juli 1547. Die Amerbachkorrespondenz, hrsg. B. J. Jenny, Bd. VI, Basel 1967, S. 507.

17 Burckhardt, Albrecht, Geschichte der Medizinischen Fakultät zu Basel 1460-1900, Basel 1917, S. 47.

${ }^{18}$ Die Amerbachkorrespondenz, hrsg. B. J. Jenny, Bd. VIII, Basel 1974, S. 216.

${ }^{19}$ Urkundenbuch der Stadt Basel, Bd.10, Basel 1908, S.380-382.

${ }^{20}$ Universitätsbibliothek Basel Ms. G II 19, fol. 255.

${ }^{21}$ Ebenda Ms. G I 26, Nr. 59.

22 Brief Hubers an Theodor Zwinger Fr. Gr. Ms. II, 23, Nr. 252.

${ }^{23}$ Ms G I 33, fol. 3.

${ }^{24}$ Portmann, M.-L., Der Schaffhauser Stadtarzt Johann Cosmas Holzach (1518-1595) und seine Schrift «Prob des Uszsatzes». Gesnerus 28 (1971), Heft 3/4, S. 147-153.

${ }^{25}$ Universitätsbibliothek Basel Ms. G ${ }^{2}$ I 17, fol. 54. 55.

${ }^{26}$ Platter, Tagebuch, S. $109 \mathrm{f}$.

${ }^{27}$ Ebenda S. 113.

${ }^{28}$ Ebenda S. 124.

${ }^{29}$ Ebenda S. 158, 262.

30 Ebenda S. 195.

${ }^{31}$ Ebenda S. 250.

32 Ebenda S.290.

33 Ebenda S. 295 f.

${ }^{34}$ Ebenda S. 299. 
${ }^{35}$ Universitätsbibliothek Basel Ms. G II 19, fol. 256.

${ }^{36}$ Ms. G II 19, fol. 257.

37 Platter, Tagebuch, S. 313.

${ }^{38}$ Ebenda S. 324.

${ }^{39}$ Ebenda S. 340.

40 Ebenda S. 364.

${ }^{41}$ Ebenda S.449. Vgl. Universitätsbibliothek Basel Ms. G' I 17, fol. 54.

42 Thommen, R., Die Rektoren der Universität Basel von 1460-1910. In: Festschrift zur Feier des 450jährigen Bestehens der Universität Basel 1910, S.479-553.

${ }^{43}$ Die Amerbachkorrespondenz, hrsg. B. J. Jenny, Bd. VI, S. 163.

${ }^{44}$ Universitätsbibliothek Basel Ms. G II 19, fol. 258.

${ }^{45}$ Dictionnaire de biographie française, Tome IX, Paris 1961, S. $1061 \mathrm{f}$.

${ }^{46}$ Brief des Gilbert Cousin an Theodor Zwinger vom 21. Oktober 1563, Universitätsbibliothek Basel Fr. Gr. Ms. I, 13, Nr. 72.

${ }^{47}$ Brief Hubers an Claude de la Baume, Erzbischof von Besançon, Universitätsbibliothek Basel Ms.Ki.Ar.18 a Bl.246-247; Fr.Gr.Ms.II, 27, Nr.15; an die Behörden von Dôle Ms. Ki. Ar. 18 a Bl.244-245; Fr. Gr. Ms.II, 27, Nr. 16.

${ }^{48}$ Pantaleon a.a. O.

\section{Summary}

Johannes Huber was first educated in Basel and Schlettstadt and then, in 1521, he matriculated in the University of Basel. He was a pupil of the famous humanist Heinrich Loriti, called Glarean. From 1525 to 1536 he studied medicine at the universities of Paris, Montpellier, and Toulouse. Having got his doctor's degree he returned to Basel where he began to practise medicine. In 1543, when Vesal was in Basel, he was rector of the University. In 1544 he became professor of physics and in 1552 he was appointed head physician of the city of Basel and professor of medicine. He deserved well by encouraging and favouring Felix Platter. Huber thus contributed to the progress of medicine.

Dr. Marie-Louise Portmann Medizinhistorische Bibliothek

Klingelbergstraße 23

CH-4031 Basel 
Beiträge zur Aargauer Geschichte

Herausgegeben von der Historischen Gesellschaft des Kantons Aargau

Willy Pfister

\section{Aargauer in fremden Kriegsdiensten}

Band 1: Die Aargauer im bernischen Regiment und in der Garde in Frankreich 1701-1792;

Die Aargauer im bernischen Regiment in Sardinien 1737-1799

304 Seiten, 7 Abbildungen, 2 Karten, 6 Grafiken. Broschiert.

Bestellnummer 0802172

Weit herum in der Schweiz wird das Aargauer Volkslied «Im Aargau sind zwöi Liebi» gesungen und gespielt. Man singt überall gerne vom Glück verliebter junger Menschen, aber unverständlicherweise und ohne Grund zieht in unserem Lied der junge Mann in fremden Kriegsdienst. Er komme wieder «über's Johr im andere Summer». Und er kehrt auch heil zurück, aber die Jungfrau hat nicht auf ihn gewartet. Seine Mutter gibt ihm auf sein Klagen die einfache Antwort: «Wärisch du deheime geblibe.» Die meisten Sänger können nicht wissen, daß unser Lied einen ganz realen Hintergrund hat und aus dem Erleben des Volkes hervorgegangen ist. Wirkliche, vielhundertfache Geschehnisse und Eindrücke haben sich zum Lied verdichtet. Es enthält genau den Ablauf einer Werbung für fremden Kriegsdienst. Da ist ein junger Mann glücklich mit seiner Jungfrau, aber unerwartet ist er einem Werber ins Garn gegangen. Die bange Frage seiner Jungfrau, wann er wiederkehre, beantwortet er im Lied dichterisch umschrieben mit «über's Johr im andere Summer», was vier Jahre Dienstverpflichtung bedeutet. Die Jungfrau wartet nicht auf einen kriegsgefährdeten Söldner. Als der heimgekehrte Soldat sich bei der Mutter über sein Unglück beklagt, gibt sie ihm deutlich zu verstehen, daß ein Mensch, der so gegen alle Vernunft gehandelt und sein Glück selbst zerstört habe, auch selbst daran schuld sei. Die Mutter spricht damit die im Volk verbreitete Meinung aus. Sie betrachtet den Kriegsdienst des jungen Mannes als Grund des Unglücks. Wer sein Glück, sein Ziel und seine Zukunft behalten möchte, müsse zu Hause bleiben: «Wärisch du deheime geblibe.»

Mit dieser Arbeit soll versucht werden, den Hintergrund des Aargauerliedes darzustellen und aufzuzeigen, wie im 18. Jahrhundert junge Menschen angeworben und dem heimatlichen Leben entführt worden sind.

Die Lokalhistoriker und Familienforscher werden besonders das Verzeichnis von über 3500 Namen mit Gewinn auswerten können. Den Militärhistorikern bietet vor allem der dritte Teil ein reiches Vergleichsmaterial.

\section{Verlag Sauerländer}

Aarau $\cdot$ Frankfurt am Main $\cdot$ Salzburg 